\title{
Secondary Ion Images of the Developing Rat Brain
}

\author{
Peter J. Todd, John M. McMahon, and Carl A. McCandlish, Jr. \\ Chemical Sciences Division, Oak Ridge National Laboratory, Oak Ridge, Tennessee, USA
}

\begin{abstract}
Secondary ion images were obtained from sections of rat brain over a 21 day postnatal period, using the intensity of $\mathrm{m} / \mathrm{z} 184$, phosphocholine. When compared with corresponding optical images of similar, but stained sections from the same animal, the secondary ion images appear to reflect less developed brains. During development, myelination occurs after axon extension. Apparently, myelination obscures the source of secondary $\mathrm{m} / \mathrm{z}$ 184, phosphatidylcholine, from the analyzing ion probe; absenting myelination, secondary ion images show no physiological features. (J Am Soc Mass Spectrom 2004, 15, 1116-1122) (c) 2004 American Society for Mass Spectrometry
\end{abstract}

I mages obtained by a variety of methods-optical, secondary electron, nuclear magnetic resonance, etc., are integral to the philosophy of all branches of medicine. Among mass spectral methods, secondary ion mass spectrometry (SIMS) and imaging matrix assisted laser desorption (iMALDI) mass spectrometry are the major players in this arena [1]. These are both emerging methods, and as such, it is necessary to establish that not only are results consistent with known mass spectrometry, but also with known biomedicine.

Secondary ions are emitted from tissue at virtually every $m / z$. We identify secondary ions by tandem mass spectrometry (MS/MS), and map the emission of ions whose structure we know. We then try to interpret the images in the context of known anatomy physiology. For example, the major peak in the secondary ion mass spectrum of almost any collection of animal cells is $\mathrm{m} / \mathrm{z}$ 184, phosphocholine. Phosphocholine is unique among secondary ions emitted from tissue in that its structure has been verified by MS/MS [2], a method permitting distinction of phosphocholine from other sources of $\mathrm{m} / \mathrm{z}$ 184 such as epinephrine. The secondary phosphocholine ion derives from the head group of phosphatidylcholine, (PC in the shorthand of biochemists) a major constituent of the animal cell membrane. Most of the outer layer of cell membranes in the brain is either phosphatidylcholine or sphingomyelin [3-5], and both compounds yield abundant $m / z$ 184. Thus, detection of phosphocholine is consistent with the chemistry of low dose or static SIMS [6], a surface analytical method, and

Published online June 7, 2004

Address reprint requests to Dr. P. J. Todd, Chemical Sciences Division, Oak Ridge National Laboratory, P.O. Box 2008, MS-6365, 1 Bethal Valley Road, Oak Ridge, TN 37831-6365, USA. E-mail: toddpj@ornl.gov the fact that most of the surface of a tissue sample is comprised of compounds yielding phosphocholine secondary ions, $m / z 184$.

The utility of SIMS for mapping phoshocholine is based on the observation that emission of phosphocholine secondary ions is heterogeneous across the tissue section surface [7]. Moreover, secondary $m / z 184$ images of adult rat brain show a direct correlation with optical images of stained tissue sections [8]. Regions of the brain containing a high density of cells such as cerebral cortex or the caudate/putamen yield an intense $\mathrm{m} / \mathrm{z} 184$ secondary ion signal, whereas acellular regions such as the corpus callosum or external capsule yield little or no $\mathrm{m} / \mathrm{z} 184$ secondary emission. In the adult, these acellular regions of the brain are very dense in cell projections (axons), many of which are enclosed by a special membrane, myelin. PC comprises $25 \%$ of glial cell plasma membrane, whereas the composition of myelin displays less than half this, but a very large component of cholesterol [9]. Thus, the significance of imaging secondary $m / z 184$ lies not in the chemistry of phosphocholine, but rather in the fact that the distribution of phosphocholine can be rationalized in the context of known cellular and tissue physiology.

Not surprisingly, secondary ion images of rat brain appear nearly identical, just as optical images of stained rat brain sections appear nearly identical. To some extent, the ion image of a rodent brain section is becoming an image reference. Still, the anatomical features of rat brain are known to arise from the process of development. Although many of the major anatomical features are present at birth, axonal projections develop significantly during the first three postnatal weeks. As the axons project out, neuroglial or glial cells associate themselves with particular axons, and wrap themselves around the axons [10], forming spiraling cellular 
sheaths about individual axons. Significantly, these processes do not occur simultaneously, but rather sequentially. By their nature, glial cells-which are not neurons-contain large amounts of fibrous proteins in their cytoplasm. As glial cells spiral around axons, the cross sections of glial cells compress. Eventually, this leads to a sheath of myelin surrounding axons. Roughly speaking, brain development consists of longitudinal growth of axons followed by radial growth of the myelin sheathes.

The purpose of the present study is not a detailed neurophysiological study of rodent development, but rather to establish correlation of secondary $\mathrm{m} / \mathrm{z} 184$ images with known development of the brain as witnessed by stained optical images. To accomplish this, brains of neonatal rats were examined at various time intervals during the first three weeks following birth. If secondary ion images of developing rat brain sections correlate with optical images of stained sections, secondary ion emission from axons is, for unknown reasons, different from emission from neurons. On the other hand, if encapsulation of axons occludes the axon cell surface, suppressing secondary ion emission, secondary ion images of developing brain must show a lag when compared with optical images of stained tissue.

\section{Experimental}

\section{Secondary Ion Mass Spectrometer}

The mass spectrometer/microprobe has been described in detail elsewhere [11]. Mass analysis was performed using a triple quadrupole mass filter, with detection by electron multiplier in analog mode. The quadrupole system suffers from loss of sensitivity at higher mass, but this is offset by the capability to perform MS/MS as needed. While time-of-flight mass spectrometers are more often employed for imaging tissue [12], performing simultaneous MS/MS and imaging with them is problematic.

Details of the primary ion gun system will be addressed elsewhere [13], and discussed here only briefly. By addition of a second primary ion gun, ion images were obtained using a primary hexabromobenzene ion beam ( $1 \mathrm{nA}, 10 \mu \mathrm{m}, 9 \mathrm{keV})$ rastered over $1 \mathrm{~cm}^{2}$. The ion beam was created by electron ionization of $\mathrm{C}_{6} \mathrm{Br}_{6}$ (Aldrich, St. Louis, MO) heated to about $100{ }^{\circ} \mathrm{C}$. The objective lens and raster system are capable of covering an area as large as $1 \mathrm{~cm} \times 1 \mathrm{~cm}$, similar to the existing $\mathrm{Cs}+$ primary ion gun [11]. Secondary ion yields from $\mathrm{C}_{6} \mathrm{Br}_{6}^{+}$appear to be greater than unity, as they are for a number of polyatomic primary ions [14] so that, at least when positive secondary ions are extracted, sample charging is not a significant factor in analysis [15]. The large sample area allows ion imaging of about $50 \%$ of a coronal section, that is, a section of a brain hemisphere. Each image is composed of 40,000 analytical sites, determined by the digital expression of the location of
Table 1. Instrumental parameters for SIMS/MS microprobe

\begin{tabular}{ll}
\hline Parameter & \multicolumn{1}{c}{ Value } \\
\hline \hline primary ion species & $\mathrm{C}_{6} \mathrm{Br}_{6}{ }^{+}$ \\
primary ion kinetic energy & $9.0 \mathrm{keV}$ \\
primary ion current & $(0.10 \mathrm{to} 1) \mathrm{nA}$ \\
primary ion spot raster & $50-100 \mu \mathrm{m}$ \\
primary ion current density & $(2.5$ to 18$) \mu \mathrm{A} / \mathrm{cm}^{2}$ \\
primary ion dose per image & $(0.2$ to 1.5$) \times 10^{12} \mathrm{ions} / \mathrm{cm}^{2}$ \\
primary ion dose/mass spectrum & .5 to $3.5 \times 10^{14} \mathrm{ions} / \mathrm{cm}^{2}$ \\
primary ion duty cycle & $(3.5 \mathrm{~ms}) /(4.5 \mathrm{~ms})$ \\
raster size & $1 \mathrm{~cm} \times 1 \mathrm{~cm}$ \\
image acquisition time & $180 \mathrm{~s}$ \\
secondary ion energy & $10 \mathrm{eV}$ \\
conversion dynode potential & $0 \mathrm{kV}$ \\
multiplier potential & $-2.1 \mathrm{kV}$ \\
data acquisition rate & $60 \mathrm{kHz}$ \\
data points per pixel & 128 \\
pixel size & $50 \mu \mathrm{m}$ \\
\hline
\end{tabular}

the primary ion spot. Operational parameters relevant to the images presented here are shown in Table 1.

\section{Animal Sample Preparation}

Sprague Dawley neonatal rats (Charles Rivers) taken from two litters were used in these studies. The first $24 \mathrm{~h}$ following birth was considered postnatal day one (PND-1). Rat pups were examined on postnatal days 1 , $3,5,7,10,12,15,21$, and 28. Adult rats were also examined and acted as controls. Rats were first anesthetized in a large glass beaker (5-10 $\mathrm{min})$ using the inhalant Metofane (methoxyflurane, Pitman-Moore). Afterwards, animals were removed from the glass beaker and intracardially perfused with buffered saline ( $\mathrm{pH}$ 7.3) followed by $2 \%$ formalin $(\mathrm{pH} 7.3)$ in accordance with the NIH Guide for Care and Use of Laboratory Animals [16] and approval of the ACUC at ORNL. Brains were removed from the skull and sectioned coronally $(50 \mu \mathrm{m})$ in $2 \%$ formalin using a Series 1000 Vibratome Sectioning System (St. Louis, MO).

\section{Section Preparation}

Sections were mounted from $2 \%$ formalin onto copper targets $(1.5 \mathrm{~mm}$ thick, $1.6 \mathrm{~cm} . \times 7.8 \mathrm{~cm})$. Prior to tissue mounting copper slides undergo conventional subbing with gelatin similar to that normally done with glass slides [8]. Gelatin coating of copper slides used for SIMS imaging allows the freshly mounted section to remain flat while in the process of air drying. Mounted sections were neither dehydrated nor cover-slipped prior to imaging. Secondary ion imaging was conducted on the same day the sections were mounted onto the copper slides. Alternate sections were mounted onto gelatinsubbed glass slides, air dried, dehydrated in graded alcohols, and stained with cresyl violet. Cresyl violet is a conventionally used niss-1 stain which binds to substances in and around the brain cell body but not to the long axonal projections, myelinated or unmyelinated, 


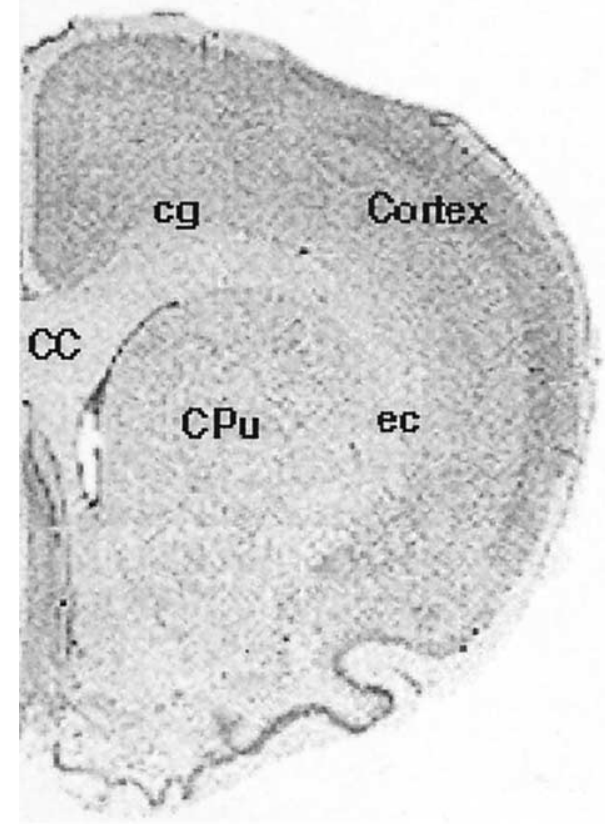

(a)

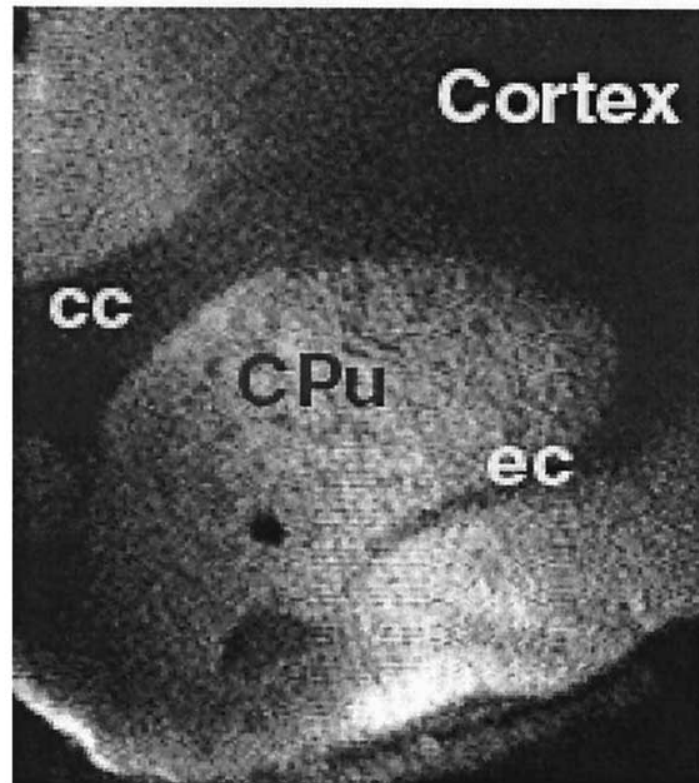

(b)

\begin{abstract}
Figure 1. Optical (a) and secondary ion image (b) of coronal sections (50 $\mu \mathrm{m})$ taken from an adult rat. Optical image is of a section stained using cresyl violet. The images reflect a field of view $1 \mathrm{~cm}$ square, and were obtained from adjacent sections, about $100 \mathrm{um}$ apart. Sections are approximately from the same frontal aspect of the brain. The dark regions observed in the ion image correspond to the axon tracts in the cresyl violet stained optical image, which do not absorb stain. In the adult these regions are quite distinct compared with the surrounding neuron-rich regions such as the cerebral cortex and caudate/putamen, which have much greater $m / z 184$ emission. Abbreviations used: CPu-caudate/ putamen, cc-corpus callosum, ec-external capsule. No abbreviation is used for the cerebral cortex; by convention, parts of the cerebral cortex are identified by relative position to other anatomical features.
\end{abstract}

which originate from the cell [17]. As a result, delineation of cell-rich regions from axonal-rich regions can readily be seen in a given section.

\section{Image Acquisition and Processing}

Digitized optical images were obtained from stained sections using VZM camera (Edmund Scientific, Tonawanda, NY) and Sherlock imaging software (Coreco Imaging, Inc., Montreal, Canada). Secondary ion image data was converted to bitmap format, and processed using Adobe Photoshop (San Jose, CA). Conversion to bitmap format necessarily involves normalization of image intensities from 0-255. Digital imaging processing was confined to cropping, rotation, and labeling. Rotation was used so that all the images were oriented in the same direction. Cropping was used to remove extraneous areas from rotated images. Labeling was added to make identification of features easier for the reader. Aside from these processes, no other image processing was used to create images presented here.

\section{Results and Discussion}

\section{Phosphocholine Distribution During Development}

Anatomical brain regions were labeled following the format of the atlas of Praximos and Watson [18]. Exam- ples of alternate adult brain sections stained with cresyl violet and imaged for $m / z 184$ are shown in Figure 1a and $b$, respectively, for comparison. Hemispherical sections are shown, as is typical with larger sections; brain halves are mirror images. As a practical matter, the field of view of the microprobe is insufficient to image an entire adult rat brain, and thus, it is possible to obtain the image of only half the brain section at a time.

Axon tracts such as the corpus callosum (cc), or external capsule (ec) stain less with cresyl violet when compared with neuron-rich regions such as the cerebral cortex and caudate/putamen $(\mathrm{CPu})$ as illustrated in Figure 1a. For the ion image, dark areas mark areas of very low phosphocholine emission, and white areas correspond to areas of intense phosphocholine emission. The caudate/putamen is comprised of cell-rich areas immediately adjacent to axonal areas, hence leading to the generalized term, striatum. The striatal appearance of the $\mathrm{CPu}$ is somewhat evident in the optical image, but fairly dramatic in the ion image, marked by small black dots. Axon track regions such as the corpus callosum and external capsule emit little $m / z 184$ when compared with the neuron-rich regions of the cerebral cortex and $\mathrm{CPu}$.

Figures 2, 3, 4, 5, and 6 show representative images obtained over the 21day postnatal study. We show the full brain section with the area marked on one side to 


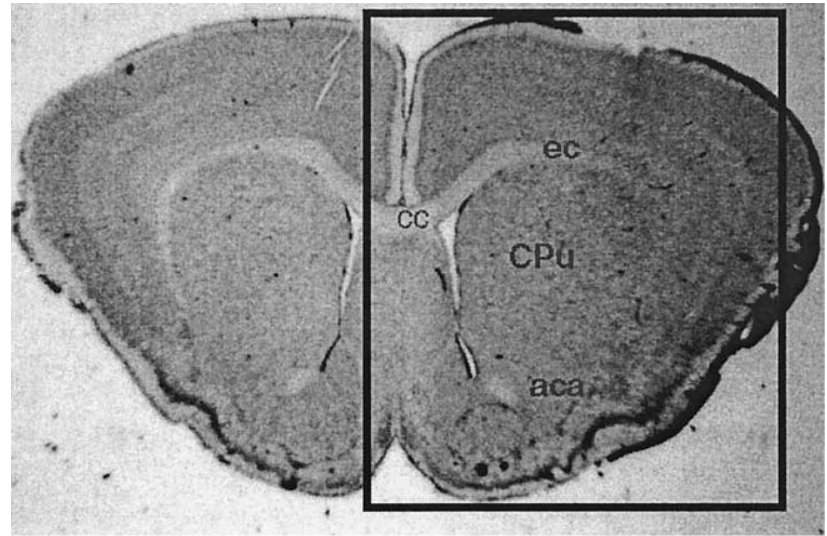

(a)

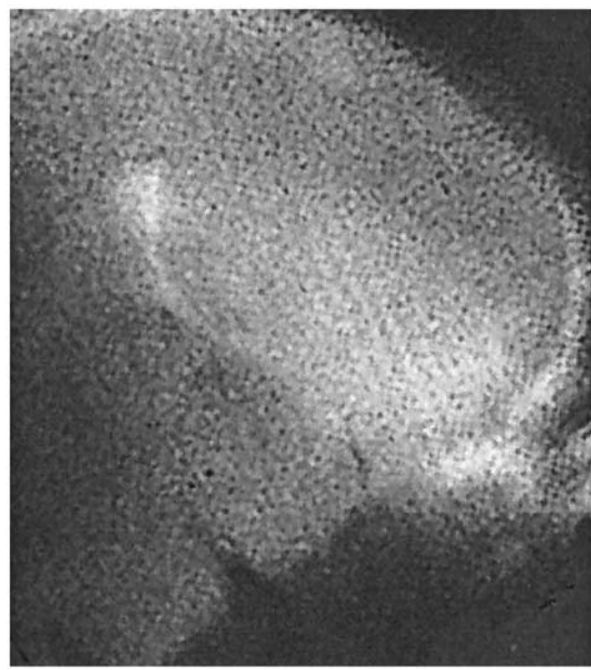

(b)

Figure 2. Optical (a) and secondary ion $(\mathrm{m} / \mathrm{z} 184)$ image $(\mathbf{b})$ of coronal section from neonatal rat one day after birth, taken from about $10 \mathrm{~mm}$ anterior of the line between ear holes. The indication interaural $10 \mathrm{~mm}$ is used in conjunction with brain atlases to identify the origin of the section relative to, in this case, the ears. Other methods are also used, mainly with adult rat brains of a particular type. For developing brains, of course, the size is generally increasing, so that assignment of particular loci is only approximate. The ion image is marked by an almost total absence of features.

indicate correspondence with the secondary ion image with the purpose of making it easier for the reader to interpret the images. Optical and $\mathrm{m} / \mathrm{z} 184$ secondary ion images obtained from postnatal day 1 rat are shown in Figure 2a and $\mathrm{b}$, respectively. The optical image shows fairly delineated features, although it is evident that the $\mathrm{CPU}$ is relatively smooth showing no evidence of striation. In contrast, the ion image is marked by the absence of any indication of development. The failure of the ion image to mimic the optical image of neonatal brain is a reproducible effect. In fact, in Figure 3, which shows optical and ion images of day 5 neonatal rat brain, a similar lack of resolution to the anatomical features of the brain is evident in the secondary ion image. The optical image shows the rapidly developing axons tracts and anatomical features.

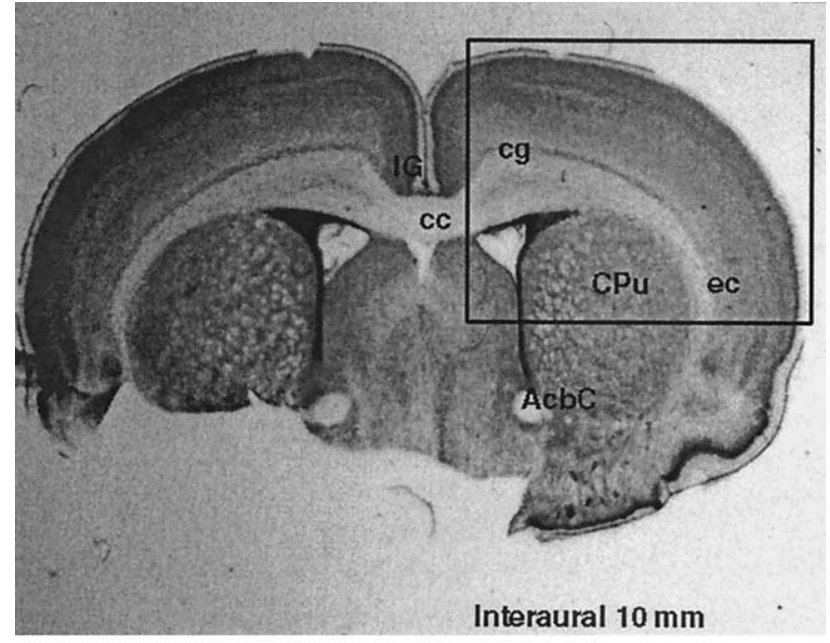

(a)

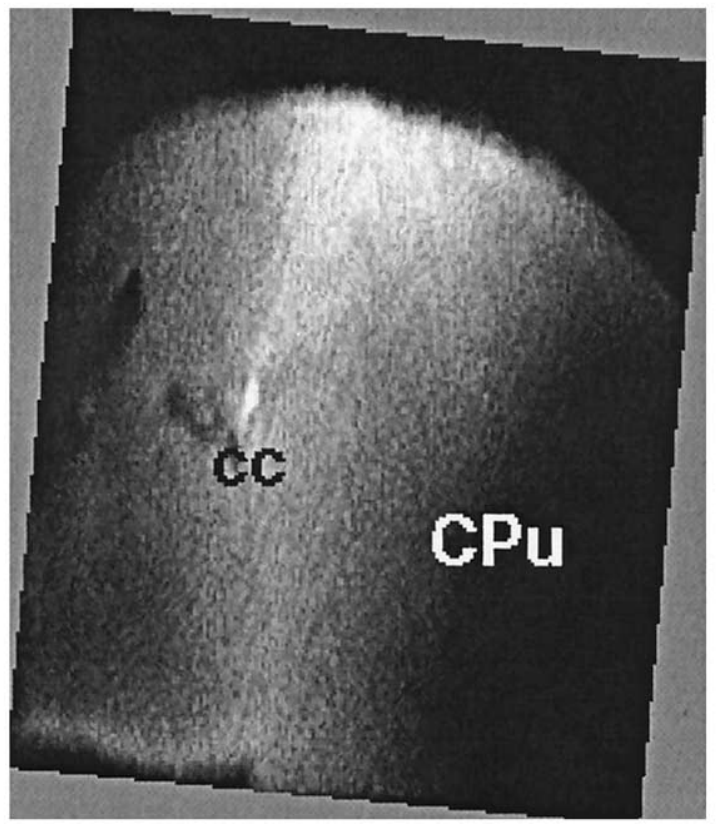

(b)

Figure 3. Optical (a) and secondary ion $(m / z$ 184) image (b) five days after birth. Despite development obvious in the stained image, no development is evident in the ion image. Slight misalignment of the target stage with respect to the mean secondary ion axis has resulted in lowered detection efficiency for the right side of the ion image.

It is not until postnatal day 10 that development appears in the secondary ion image. While the optical image in Figure 4a shows continuing development, the ion image of Figure $4 \mathrm{~b}$ shows what appears to be the beginning of the corpus callosum and its extension to the external capsule. In addition, the anterior commissure (aca) is readily apparent. These features are well developed in both optical and ion images by postnatal day 15, as shown in Figure 5.

In Figure 6, we show a slightly different (more anterior) section of the brain postnatal day 21, where the external capsule of the corpus callosum is clearly 


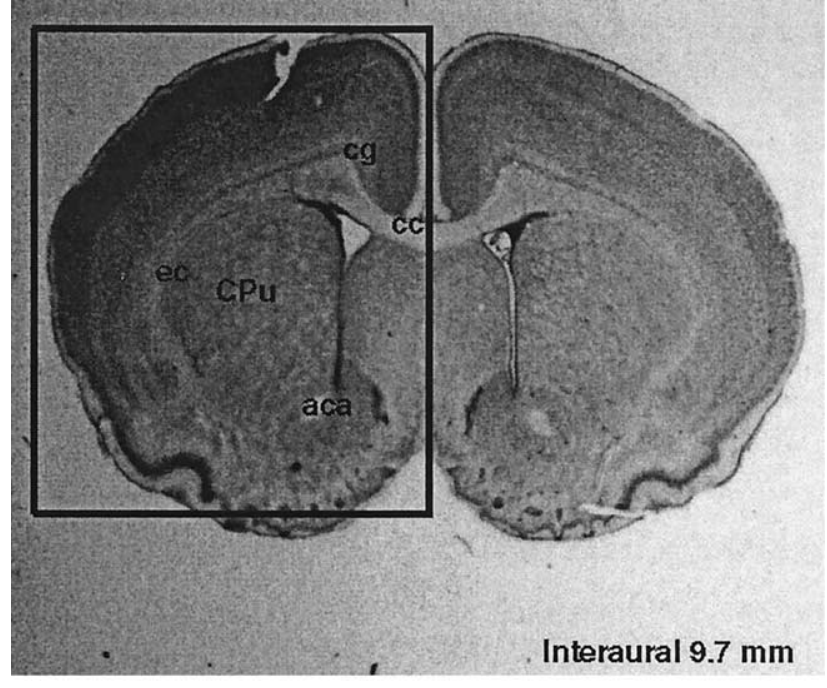

(a)

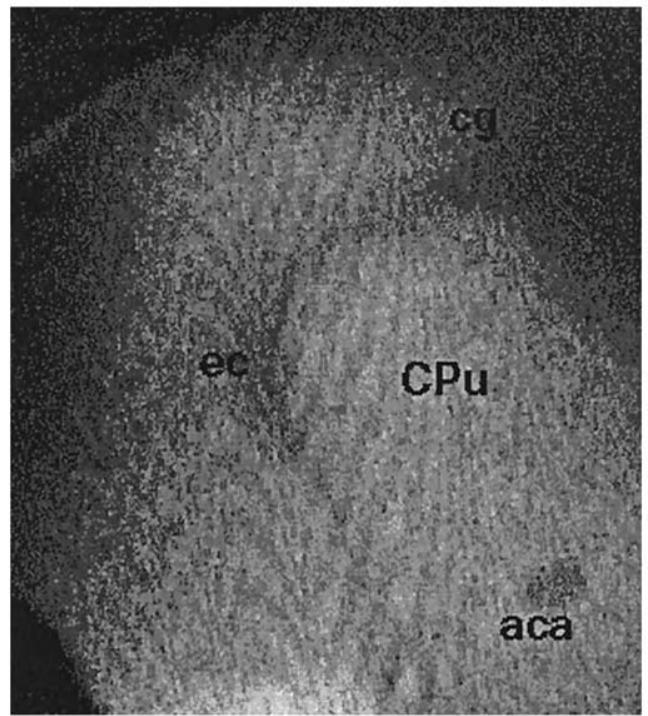

(b)

Figure 4. Optical (a) and secondary ion $(\mathrm{m} / \mathrm{z} 184)$ image $(\mathbf{b})$ ten days after birth. At this stage the myelination of the corpus callosum and its external capsule finally become evident in the ion image.

evident in both optical and ion images. These images demonstrate correlation between ion and optical images of the developing rat brain at areas other those than shown in Figures 2-5.

Axon tracts do not absorb stain whether they are myelinated or not, while cell rich areas of the brain absorb significant stain. In the adult rat, axon tracts are evident in optical images of stained brain sections for this reason. Secondary phosphocholine images, on the other hand, indicate the extent of myelination, not the presence or absence of axons. In the adult rat, optical and secondary ion images correlate exactly because axons are all myelinated. From neurophysiology, myelination in the brain is known to follow the early extension of axons from brain cells [19]. Optical and secondary ion images of the developing rat brain are

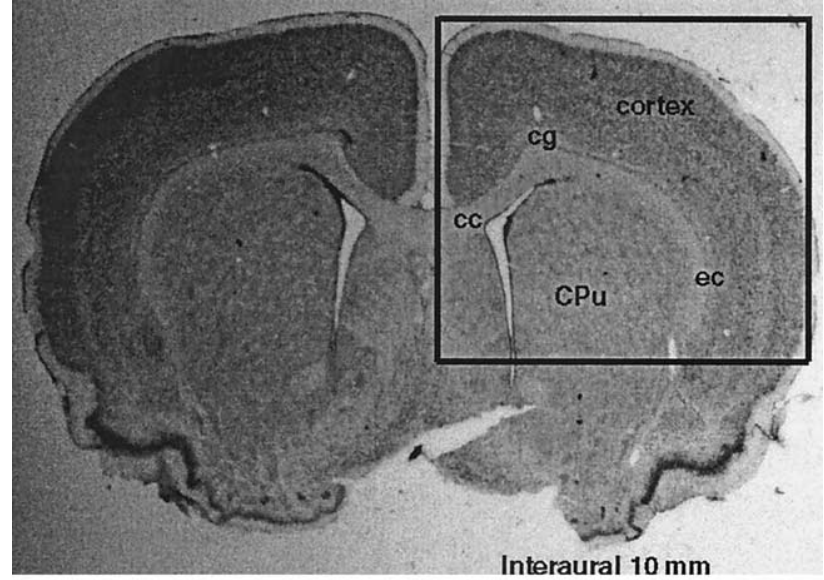

(a)

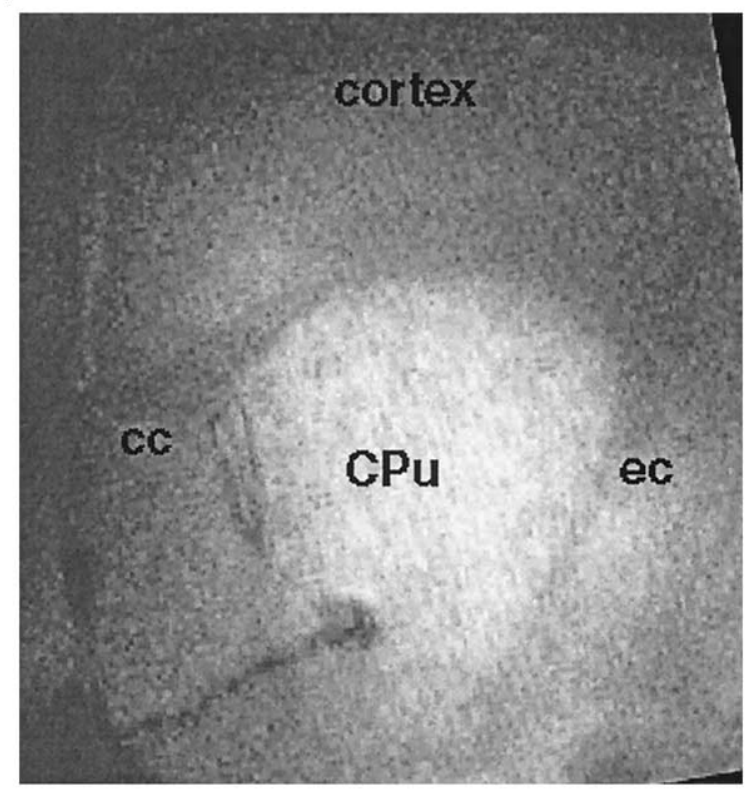

(b)

Figure 5. Optical (a) and secondary ion $(\mathrm{m} / \mathrm{z}$ 184) image (b) 15 days after birth. The ion image shows evidence of extensive myelination in appropriate areas.

consistent with this knowledge. Development of anatomical features, as evident in secondary ion images, appears to occur at a later date than that evident in optical images.

Myelination of axons is known to be critical in electrical communication between cells, anatomical stability, and cell nourishment [20,21]. Breakdown in the myelin sheathing and the organizing glia cells leads to a biochemical and morphologic cascade of detrimental events. The significance of the results presented here lies in the fact that the suppression of secondary phosphocholine ions by myelination is consistent with known brain development: myelination follows axon extension.

\section{Conclusions}

A number of features must be demonstrated for an analytical imaging method to be of use to the biomed- 


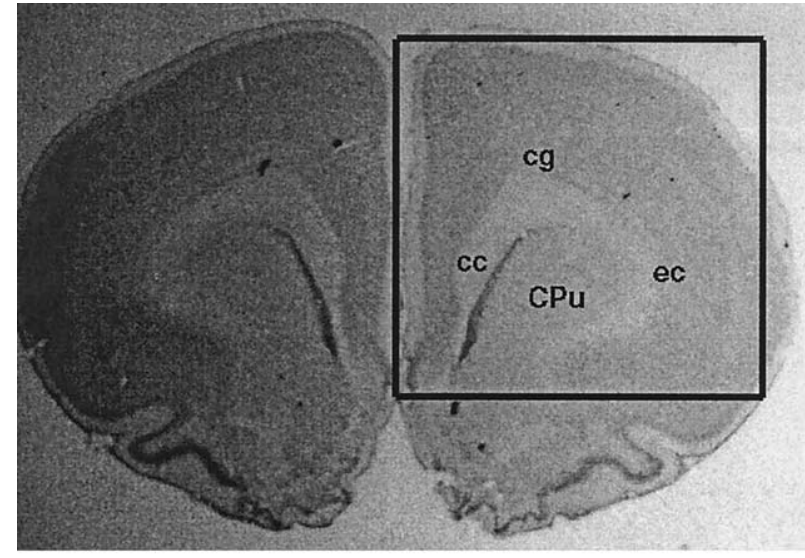

(a)

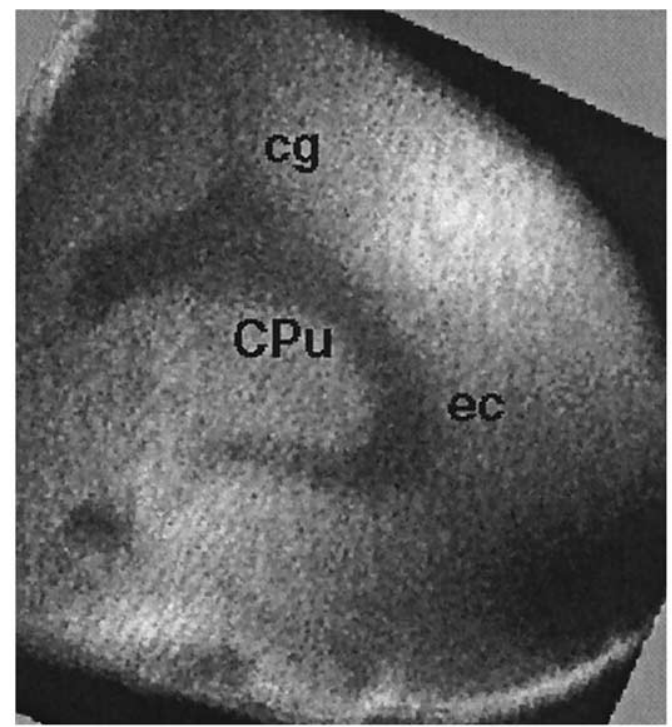

(b)

Figure 6. Optical (a) and secondary ion ( $m / z$ 184) image (b) 21 days after birth. The ion image shows complete myelination of the corpus callosum and its external capsule, and is taken of the brain anterior about $1 \mathrm{~mm}$ to the images shown in Figure 5. By 21 days, development of the rat brain is normally considered complete.

ical community. Among them is the requirement that the images and other data obtained must be consistent with known biomedicine. To the extent examined, this feature is now established for the secondary ion mass spectrometry of $m / z 184$.

It should be reiterated that the work reported here is not meant to represent any kind of detailed study of rat brain development. Future progress will be threefold: (1) To study other lipids and their distribution in both the outer and inner bilayer membrane, (2) to map spatially their fatty acid chains, and (3) to improve the spatial resolution of the molecular primary ion beam to better than $1 \mathrm{um}$. A previous report describes a method using SIMS in which the surface of a tissue sample may be bombarded by primary ions to "uncover" deeper membrane strata [22], and we anticipate similar enhanced capability [14] with our molecular primary ion source [13]. Theoretically, this technique could be used to "sputter away" or remove the outer monolayer of a membrane exposing the inner monolayer for surface analysis. Research in areas investigating models for degenerative diseases such as Alzheimer's disease or multiple sclerosis indicate that the alteration of membrane lipids are intimately involved in functions such as signal transduction and protein metabolism in the brain [21, 23, 24]. To create distributional maps of various lipids and their fatty acid chains as well as proteins would hopefully enhance an understanding of brain development and disease, and assist diagnostic procedures for any deviation from the normal physiologic events occurring during development and adulthood.

\section{Acknowledgments}

The authors are indebted to Dr. Sanford P. Markey and Professor J. T. Brenna for many helpful discussions. This research was sponsored by grant RO1-GM41617 from the National Institutes of Health. CAM gratefully acknowledges receipt of a postdoctoral fellowship sponsored by Oak Ridge Associated Universities. Oak Ridge National Laboratory is managed and operated by UTBattelle, LLC, for the U.S. Department of Energy under contract DE-AC05-00OR22725.

\section{References}

1. Todd, P. J.; Schaaff, T. G.; Chaurand, P.; Caprioli, R. M. Organic Ion Imaging of Biological Tissue with Secondary Ion Mass Spectrometry and Matrix-Assisted Laser Desorption/ Ionization. J. Mass Spectrom. 2001, 36, 355-369.

2. McMahon, J. M.; Short, R. T.; McCandlish, C. A.; Brenna, J. T.; Todd, P. J. Identification and Mapping of Phosphocholine in Animal Tissue by Static Secondary Ion Mass Spectrometry and Tandem Mass Spectrometry. Rapid Commun. Mass Spectrom. 1996, 10, 335-340.

3. Bretscher, M. S. The Molecules of the Cell Membrane. Sci. Am. 1985, 253, 122-131.

4. Murphy, R. C. Mass Spectrometry Of Lipids, Vol. VII; Plenum Press: New York, 1993; pp 213-268.

5. Vanhoeve, R. P.; Emmelot, P. Studies on Plasma-Membranes. 18. Lipid Class Composition of Plasma-Membranes Isolated from Rat and Mouse Liver and Hepatomas. J. Membr. Biol. $1972,9,105-126$.

6. Benninghoven, A.; Rudenauer, F. G.; Werner, H. W. Secondary Ion Mass Spectrometry; John Wiley and Sons: New York, 1987; pp 671-752.

7. Todd, P. J.; McMahon, J. M.; Short, R.T.; McCandlish, C. A. Organic SIMS of Biologic Tissue. Anal. Chem. 1997, 69, 529A535A.

8. McCandlish, C. A.; McMahon, J. M.; Todd, P. J. Secondary Ion Images of the Rodent Brain. J. Am. Soc. Mass Spectrom. 2000, 11, 191-199.

9. Bradford, H. E. Chemical Neurobiology; W. H. Freeman and Co.: New York, 1986; p 29.

10. Crang, A. J.; Rumsby, M. G. Intrinsic Fluorescence of Isolated Central Nervous System Myelin Sheath Preparations. Biochem. J. 1979, 177, 739-745.

11. Grimm, C. C.; Short, R. T.; Todd, P. J. A Wide-Angle Secondary Ion Probe for Organic Ion Imaging. J. Am. Soc. Mass Spectrom. 1991, 2, 362-371.

12. Pacholski, M. L.; Winograd, N. Imaging with Mass Spectrometry. Chem. Rev. 1999, 99, 2977-3005.

13. Todd, P. J., unpublished. 
14. Weibel, D.; Wong, S.; Lockyer, N.; Blenkinsopp, P.; Hill, R.; Vickerman, J. C. A C-60 Primary Ion Beam System for Time of Flight Secondary Ion Mass Spectrometry: Its Development and Secondary Ion Yield Characteristics. Anal. Chem. 2003, 75(7), 1754-1764.

15. Appelhans, A. D; Dahl, D. A.; Delmore, J. E. Neutralization of Sample Charging in Secondry Ion Mass Spectrometry via a Pulsed Extraction Field. Anal. Chem. 1990, 62(15), 16791686.

16. Guide for the Care and Use of Laboratory Animals; U.S. Department of Health and Human Services. Public Health Service, National Institutes of Health. NIH Publication No. 86-23, Revised 1985. ORNL Protocol 0224.

17. Kelly, D. E.; Wood, R. L.; Enders, A. C. Bailey's Textbook of Microscopic Anatomy; William and Wilkins: Baltimore, 1984; $\mathrm{p}$ 315.

18. Praxinos, G.; Watson, C. The Rat Brain in Stereotaxic Coordinates, 2nd ed.; Academic Press: San Diego, 1986; p ix.

19. Webster, H. D.; Martin, R.; O'Connell, M. F. Relationships between Interphase Schwann Cells and Axons before Myeli-
nation-Quantitative Electron-Microscopic Study. Dev. Biol. 1973, 32, 401-416.

20. Quarles, R. H.; Farrer, R. G.; Yim, S. H. In Cell Biology and Pathology of Myelin; Juurlink, B. H.; Devon, R. M.; Doucette, J. R.; Nazarali, A. J.; Schreyer, D. J.; Verge, V. M., Eds.; Plenum: New York; 1973; p 5.

21. Blakemore, W. F.; Crang, A. J.; Keirstead, H. S.; Franklin, R. J. M. Recent Insights into the Cellular Biology of Rremyelination: Im- plications for Multiple Sclerosis. Cell Biology and Pathology of Myelin; Kuurlink, B. H.; Devon, R. M.; Doucette, J. R.; Nazarali, A. J.; Schreyer, D. J.; Verge, V. M., Eds.; Plenum Press: New York, 1997; p 235.

22. McMahon, J. M.; Dookeran, N. N.; Todd, P. J. Organic Ion Imaging Beyond the Limit of Static SIMS. J. Am. Soc. Mass Spectrom. 1995, 6, 1047-1058.

23. Gustavsson, L.; Alling, C. Effects of Chronic Ethanol Exposure on Fatty-Acids of Rat-Brain Glycerophospholipids. Alcohol 1988, 6, 139-146.

24. Prasad, M. R.; Lovell, M. A.; Yatin, M.; Dhillon, H.; Markesbery, W. R. Regional Membrane Phospholipid Alterations in Alzheimer's Disease. Neurochem. Res. 1998, 23, 81-88. 